\title{
A New Alternative Method for the Generation of Acoustic Filters, Modulating Acoustic Impedance: Theoretical Model
}

\author{
Jesús Madrigal-Melchor'1, Agustín Enciso-Muñoz, David Armando Contreras-Solorio1, \\ Xochitl Saldaña-Saldaña ${ }^{2}$, Raúl Alberto Reyes-Villagrana ${ }^{3 *}$ \\ ${ }^{1}$ Unidad Académica de Física, Universidad Autónoma de Zacatecas, Zacatecas, México \\ ${ }^{2}$ Instituto de Física, Benemérita, Universidad Autónoma de Puebla, Puebla, México \\ ${ }^{3}$ CONACYT-Universidad Autónoma de Chihuahua, Chihuahua, México \\ Email: *rareyesvi@conacyt.mx
}

How to cite this paper: Madrigal-Melchor, J., Enciso-Muñoz, A., Contreras-Solorio, D.A., Saldaña-Saldaña, X. and Reyes-Villagrana, R.A. (2017) A New Alternative Method for the Generation of Acoustic Filters, Modulating Acoustic Impedance: Theoretical Model. Open Journal of Acoustics, 7, 39-51.

https://doi.org/10.4236/oja.2017.73005

Received: June 9, 2017

Accepted: August 1, 2017

Published: August 4, 2017

Copyright $\odot 2017$ by authors and Scientific Research Publishing Inc. This work is licensed under the Creative Commons Attribution International License (CC BY 4.0).

http://creativecommons.org/licenses/by/4.0/

\begin{abstract}
Using the transfer matrix method we calculate the frequency dependence of the transmission of longitudinal elastic waves for a layered structure where the specific acoustic impedance of the layers with odd numbering follows a Gaussian distribution, while the inserted even layers have the same impedance as the propagation medium. The structure presents intervals of low-pass, bandstop, and band-pass. The characteristics of the bands depend on the number of layers, on the contrast between the maximum and minimum impedances of the structure, and on the ratio of the width of the inserted layers to the width of the layers with a Gaussian distribution of impedances.
\end{abstract}

\section{Keywords}

Acoustic Transmission, Acoustic Impedance Modulation, Gaussian Profile

\section{Introduction}

Since many decades ago, the search for energy, electronic, optical and acoustic filters is an active field. In the area of acoustic, studies by Rayleigh opened an interest in the exploration of sound [1]. Pupins, Cambell and Wagner made the first studies to transmit information, where they proposed a transmission line and filtered the signals through simple configuration, known as $\mathrm{T}$ and $\pi$ [2] [3] [4]. Later, the work development by Stewart in 1922 [5] [6], began the study by acoustic filters. Stewart focused in the study on the relationship of the acoustic transmission between different media and applied the concepts of acoustic im- 
pedance, thus and analogy related to electrical circuits [7] [8]. Subsequently there was a series of works such as Peacock, Mason and Lindsay with which deepened and broadened the investigation of acoustic filters, then using the broad term studies of transmission line and varying conditions [9]-[14]. Today there extended literature on these issues. However, they have combined a group of applications, not only in acoustic but also in other research field, like signal processing, telecommunications, medicine, etc. [15]-[63]. In particular, there are proposals of energy band-pass filters using quantum superlattices with Gaussian potential profile [64] [65]. These structures allow the incident electrons to be nearly totally transmitted when the impinging energy is in the stop-band. The characteristics of the bands can be adjusted modifying the parameters of the superlattice and of Gaussian distribution. On the other hand, following the preceding idea, there is also a proposal of a multilayer optical structure where refractive index varies according to the envelope of Gaussian functions [66]. This structure acts as an omnidirectional mirror. For sound, the difference between acoustic impedance values between two media causes reflection at the interface. We propose in this work a multilayer acoustic filter where the specific acoustic impedance of the layers with odd numbering is modulated by a Gaussian functions. The acoustic impedance of the inserted provides a slow impedance for the layers, which can improve the transmission of the structure. We make a theoretical study of the transmission for this structure following a formalism of transfer matrix used for electromagnetic waves, which we have adapted to acoustic waves [67]. The $100 \%$ reflectivity and practically $100 \%$ transmission can be obtained when the frequency lies within the respective bands. The bands are flat and their positions and bandwidths are adjustable. This type of filter can be constructed experimentally using layers with composite materials where the acoustic impedance can be tailored by varying the volume fractions of the components in the composite [68] [69]. Another possible way to construct this filter is by using layers of porous silicon. This material has been widely used for the fabrication of optical devices, including optical filters where the refractive index can be varied through a variation of the porosity [66]. There are also studies of the variation of acoustical properties, including the acoustical impedance, due to the variation of the porosity [70] [71]. Recently, acoustic multilayer mirrors have been made using porous silicon [72]. In previous work, studies on acoustic, electronic and optical properties were made [73] [74] [75]. In this work we propose an alternative for generating acoustic filter from the modulation of the acoustic impedance and it is an effective method for making a better coupling of the acoustic impedances.

\section{Theoretical Model and Calculation Method}

For calculate the transmittance, we use the theory of references [67] [76] [77]. We consider a structure of $\mathrm{N}$ plane multilayers. The layers are perpendicular to the x axis. Each j-layers has a width $d_{j}$ and acoustic $Z_{j}$ given by 


$$
Z_{j}=\rho_{j} c_{j}
$$

where $\rho_{j}$ and $c_{j}$, the density and the acoustic longitudinal speed for the $j$-material, respectively. We consider longitudinal elastic plane waves propagating in the $\mathrm{X}-\mathrm{Z}$ plane, coming from left in a propagation medium with impedance $\mathrm{Z}_{\mathrm{PM}}$. The plane wave is incident on the structure of $\mathrm{N}$ plane multilayers. At the right side of the structure, the propagates in a medium PM with impedance $Z_{\mathrm{PM}}$. We can write the wave function for each layer in the following form,

$$
P_{j}=A_{j} \mathrm{e}^{i\left(\overrightarrow{\boldsymbol{k}}_{j} \cdot \overrightarrow{\boldsymbol{r}}-\omega t\right)}+B_{j} \mathrm{e}^{i\left(\overrightarrow{\boldsymbol{k}}_{j}^{\prime} \cdot \overrightarrow{\boldsymbol{r}}-\omega t\right)}
$$

where $P_{j}$ presents the propagating wave pressure perturbation. The first and the second terms on the right-hand side Equation (2) represent propagation to the right and to the left, respectively, i.e. the forward and the backward waves. The index $j=0$ represents the propagation medium at the left of the structure with impedance $\mathrm{Z}_{\mathrm{PM}}$. In the medium $\mathrm{PM}$ with $j=N+1$ we consider only propagation to the right, consequently $B_{S}=0 . k_{j}$ and $k_{j}^{\prime}$ are the forward and backward wave vectors for medium $j, t$ is the time, $\omega$ the angular frequency, and $i$ the imaginary unit. A solid can support both longitudinal and transverse elastic wave, and a fluid only transmits elastic longitudinal waves. If a longitudinal wave in a fluid is incident obliquely on the interface with a solid, both type of waves can be transmitted in the solid. However, at normal incidence, the character of the longitudinal wave is preserved, without generation of transverse wave in the solid. Then, for oblique incidence, our theory is valid only for fluid layers. If wave is incident at an angle $\theta_{0}$ with the normal to the structure, law of Snell gives,

$$
\frac{1}{c_{0}} \sin \theta_{0}=\frac{1}{c_{1}} \sin \theta_{1}=\cdots=\frac{1}{c_{j}} \sin \theta_{j}=\cdots=\frac{1}{c_{N}} \sin \theta_{N}=\frac{1}{c_{S}} \sin \theta_{S}
$$

The wave propagation from medium 0 to medium $S$ through the multilayer structure is described propagation wave by,

$$
\left[\begin{array}{l}
A_{0} \\
B_{0}
\end{array}\right]=\left[\begin{array}{ll}
M_{11} & M_{12} \\
M_{21} & M_{22}
\end{array}\right] \cdot\left[\begin{array}{c}
A_{S} \\
0
\end{array}\right]
$$

where the (2X2) transfer matrix is given by [67],

$$
\left[\begin{array}{ll}
M_{11} & M_{12} \\
M_{21} & M_{22}
\end{array}\right]=D_{0}^{-1}\left[\prod_{j=i}^{N} D_{j} P_{j} D_{j}^{-1}\right] D_{S}
$$

The matrix $D_{j}$ is called the dynamical or transmission matrix, and arises from the continuity conditions on the pressure and the displacement normal to the interface between media $j-1$ and $j$. The matrix $P_{j}$ is the kinematical or propagation matrix inside the $j$ layer. If it supposed that the media are lossless, the matrix are given by,

$$
D_{j}=\left[\begin{array}{cc}
1 & 1 \\
\frac{\cos \theta_{j}}{Z_{j}} & -\frac{\cos \theta_{j}}{Z_{j}}
\end{array}\right]
$$




$$
P_{j}=\left[\begin{array}{cc}
\exp \left(i k_{j} d_{j} \cos \theta\right) & 0 \\
0 & \exp \left(-i k_{j} d_{j} \cos \theta\right)
\end{array}\right]
$$

where $d_{j}$ is width layer in the structure. We define the transmission coefficient $T$ as the transmitted power by the waves through the structure [78], normal to the structure, divided by the power of the incident waves, normal to the structures. It is given in terms of the transfer matrix by,

$$
T=\left|\frac{\left\langle P_{t}\right\rangle \cdot \hat{n}}{\left\langle P_{i}\right\rangle \cdot \hat{n}}\right|
$$

where $P_{t}$ is the vector of transmitted power, $P_{i}$ the vector of incident power, and $\hat{n}$ the unit vector normal to the structure. The angle brackets denote average over time. $P$ is similar to the Poynting vector in electromagnetism, which is given by $\boldsymbol{S}=\boldsymbol{E} \times \boldsymbol{H}$, where $\boldsymbol{S}$ is the vector of Poynting, $\boldsymbol{E}$ is the vector of the electric field and $\boldsymbol{H}$ is the vector of the magnetic field intensity, units are $\left(\mathrm{W} / \mathrm{m}^{2}\right)$. Similarly, the acoustic Poynting vector is given by $\boldsymbol{\Xi}=-\boldsymbol{T} \boldsymbol{v}$, where $\boldsymbol{\Xi}$ is the acoustic Poynting vector, $\boldsymbol{T}$ is the stress to which the studied material is subjected and $\mathrm{v}$ is the particle velocity, the units of the acoustic Poynting vector are $\left(\mathrm{W} / \mathrm{m}^{2}\right)$ [79]. Its temporal average is given by [68],

$$
\langle P\rangle=\frac{1}{2} \frac{A^{2}}{Z} \hat{u}
$$

here, $A$ is the amplitude of the pressure wave and $\hat{u}$ the unit vector in the direction of propagation of the wave. The transmission $T$, in terms of the transfer matrix, is given by,

$$
T=\frac{Z_{0} \cos \theta_{S}}{Z_{S} \cos \theta_{0}}\left|\frac{1}{M_{11}}\right|
$$

The reflectance $R$ is given by,

$$
R=T-1
$$

The specific acoustic impedance for the layers with odd numbers (Gaussian layers) in modulated by the Gaussian function,

$$
Z(x)=\left(Z_{\text {max }}-Z_{\text {min }}\right) \mathrm{e}^{\left(\frac{-\left(x-x_{0}\right)^{2}}{\sigma^{2}}\right)}+Z_{\text {min }}
$$

where $Z_{\max }$ is the maximum impedance for the Gaussian values and $Z_{\min }$ is the impedance of the medium where the structure is situated, which is the same as the medium of the inserted even layers.

\section{Results and Discussion}

We consider that the structure is located in a propagation medium such as water, because later in the experimental stage, it can minimize signals or unwanted information (noise), compared to use in the air, as propagation medium. The following parameters are proposed with a minimum and maximum impedance. The proposed material is a composite, which has a range of minimum and 
maximum acoustic impedance, which are, $Z_{0}=Z_{\min }=1.509$ MRayls, $\rho_{0}=\rho_{\min }=$ $1000.0 \mathrm{~kg} / \mathrm{m}^{3}, c_{0}=c_{\min }=1509.0 \mathrm{~m} / \mathrm{s}, Z_{\max }=3.5$ MRayl, $\rho_{\max }=1500.0 \mathrm{~kg} / \mathrm{m}^{3}, c_{\max }$ $=2333.0 \mathrm{~m} / \mathrm{s}$. The total thickness of the structure is fixed, with a value $\Delta=1 \mathrm{in}$ arbitrary units. For the Gaussian function, we use a value of $\sigma=\Delta / 4$, which for our calculations gives an efficient transmission. For the calculations, it is necessary to know for each value of $Z$ calculated by Equation (12), the corresponding values of $\rho$ and $c$ for the Gaussian layers. For that purpose, we also make a Gaussian interpolation for $\rho$ between the values of $\rho_{\max }$ and $\rho_{\min }$, and find the corresponding values of $c$ using Equation (1). The Gaussian layers have a width $d_{G}$ and the inserted layers have a width $d_{I}$. The impedance profile of the structure is show schematically in Figure 1.

The spectrum of allowed frequencies for an acoustical multilayer structure consists of quasi-bands of discrete values of eigenfrequencies, separated by gaps or stop-bands, where there is no transmission of sound [80].

We present in Figure 2 the transmittance for normal incidence for a structure with a total of 45 layers, where 23 follow the Gaussian profile, with three different values of the ratio $d_{I} / d_{G}=1,3,5$ as a function of $w_{D} / c_{\text {water }}$, where $c_{\text {water }}$ is the speed of sound for water, when the ratio $d_{I} / d_{G}$ increases, there is better transmission in the pass-band, their width increases and size of the stop-bands decreases.

The reason of this improvement of the transmission, is that the fraction filled by Gaussian layers which have larger values of impedance than the water.

In Figure 3 we make a comparison of the transmission spectra between a structure with Gaussian profile of impedances and a structure with a regular profile, where the layers with odd numbers have constant value of impedance $Z$ $=3.0$ MRayls. The structure with regular profile of impedances has the expected gaps of frequencies (stop-bands) but it does not have flat pass-bands as the structure with Gaussian profile. The oscillations that occur in the transmission spectrum for the regular structure (a), correspond to the eigenfrequencies. We observe 23 oscillations in each quasi-band, which correspond to the 23 layers that have the Gaussian profile (b).

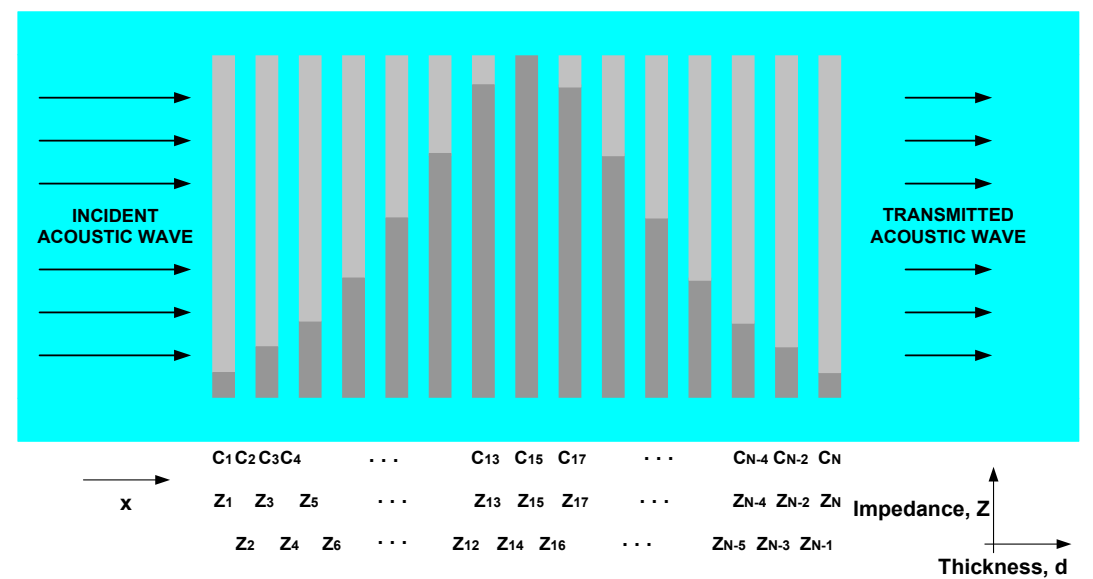

Figure 1. The impedance profile of the structure, with the gray layer follows a Gaussian profile and has a width $d_{G}$ 

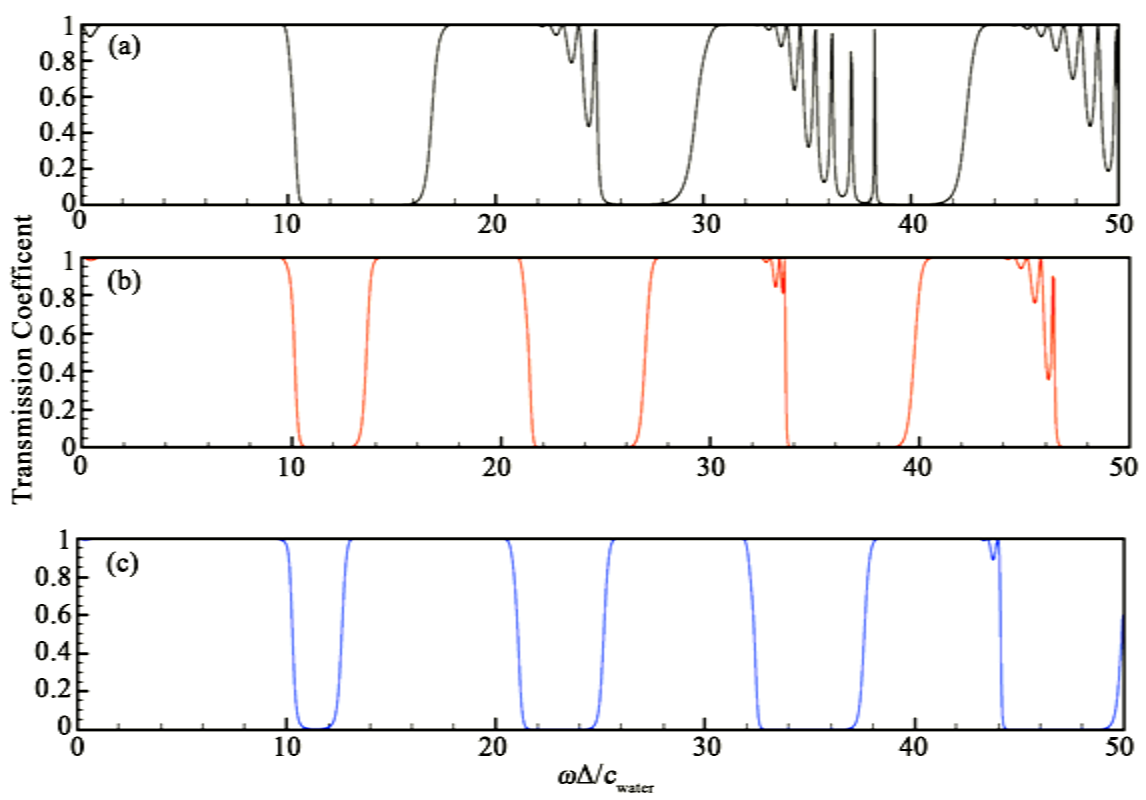

Figure 2. Transmission coefficient for normal incidence for a structure with a total of 45 layers, with $Z_{\min }=1.509$ MRayls and $Z_{\max }=3.5$ MRayls and where 23 follow the Gaussian, with three different values of the ratio (a) $d_{I} / d_{G}=1$, (b) $d_{I} / d_{G}=3$ and (c) $d_{I} / d_{G}=5$.

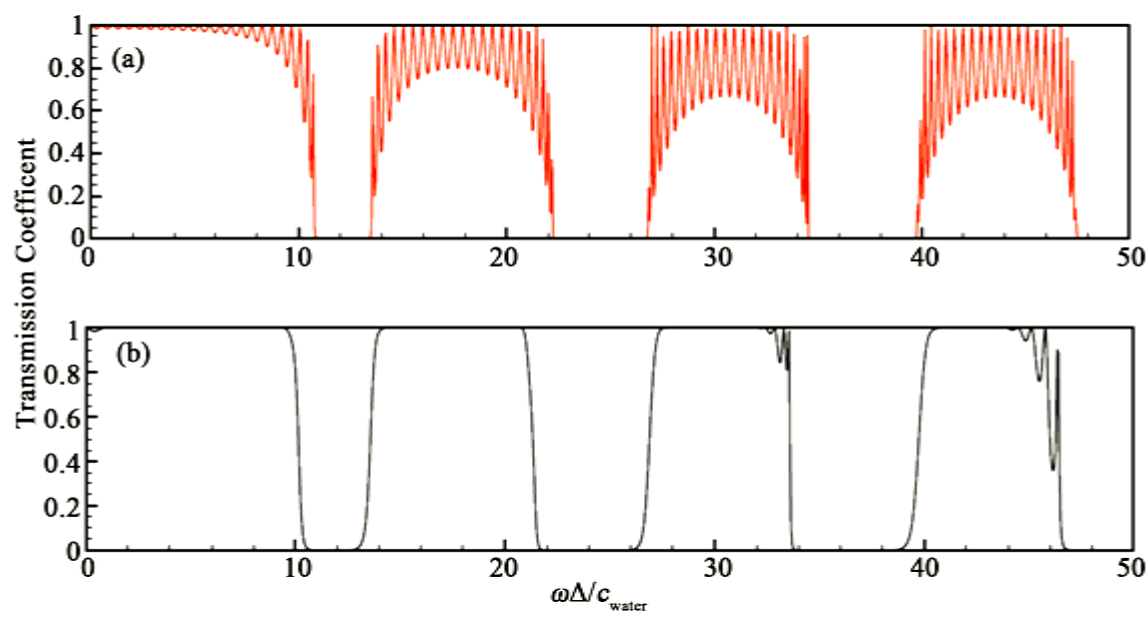

Figure 3. Transmission coefficient for a (a) regular profile and (b) Gaussian profile with $d_{I}$ $=3 d_{G}$ and 45 total layers.

The transmission in the pass-bands for the structure with regular profile is poorer due to the more abrupt change of impedances. A structure with regular profile can work as an acoustic mirror, but it is bad as an acoustic filter.

In the Figure 4 we show the spectra of transmission for two structures with different number of layer. If we put more layers in the structure, the bands move upwards. This behaviour is similar to that of the quasi-bands of energies for an electron is a superlattice when the wells and barriers are narrower.

Also, the pass-bands are wider because the number of eigenfrequencies increases. At the same time, the reflectance for the stops-bands is improved due to 


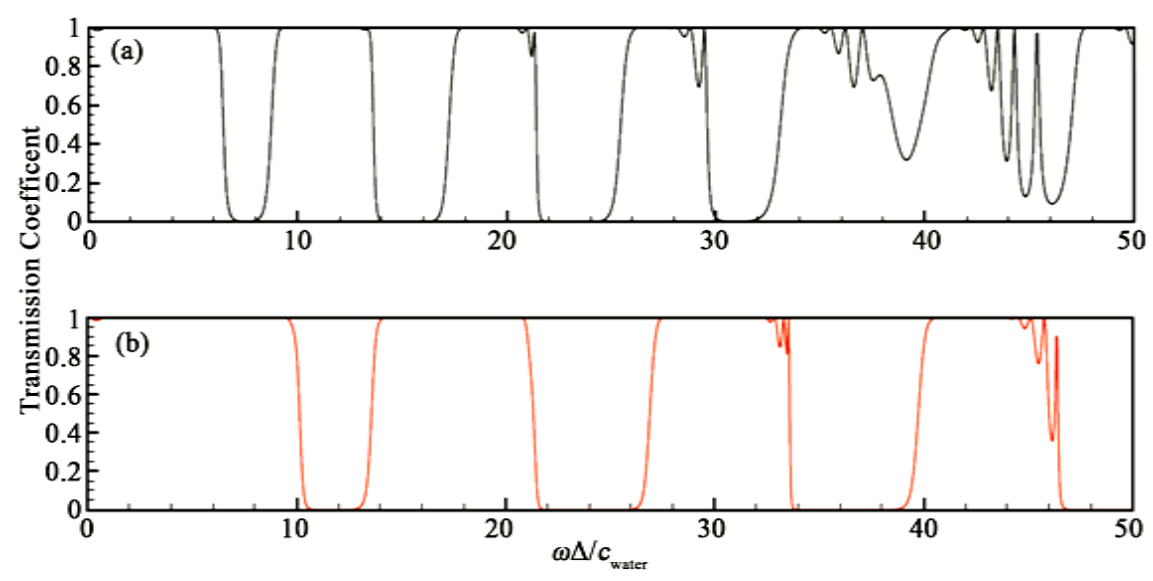

Figure 4. Transmission coefficient for a ratio $d_{G} / d_{I}=3$ the structure with (a) 29 layers and (b) 45 layers.

the fact that there are more layers with large impedance. We present in Figure 5 the transmittance for two values of $Z_{\max }=3.5$ y 7.5 MRayls. When the difference between $Z_{\min }$ and $Z_{\max }$ is larger, the pass-bands area narrower because there is a more abrupt change for the impedance of the Gaussian layers, which causes more reflection. We stress that the positions of the pass-bands and widths of the bands can be adjusted changing the parameters of the structure.

Finally in Figure 6 we show the transmittance of oblique incidence for four values of incidence angle $(0,45,70$ and 80$)$ for the structure with 45 layers. When the angle of incidence increases the bands move towards intervals of higher frequencies and the transmission is poorer, as expected.

At the same time, the low-pass band becomes wider. About 80 the transmission practically disappears. We emphasize our calculations for oblique incidence is valid only for fluid layers.

\section{Conclusions}

Using a method of transfer matrix for electromagnetic waves, we have made studies in order to propose a layered acoustic filter where the characteristic impedance of the layers with odd numbers follows a Gaussian distribution and the inserted layers with even number have a constant value of acoustic impedance. Adjustable flat transmission bands and reflection bands are obtained by properly choosing the structure parameters, when a longitudinal plane wave of sound is incident on the layered structure, is practically transmitted totally if the frequency lies in a pass-band and fully reflected if the frequency lies in a stop-band. These properties have a wide area of application, such as in acoustic mirrors and filters. The latter allows to select specific frequency ranges to pass through them, for example in applications of medical ultrasound and the photoacoustic spectroscopy; also in the exploration and study of food to meet their properties and to apply the acoustic tools for food processing, as is the high intensity ultrasound. 


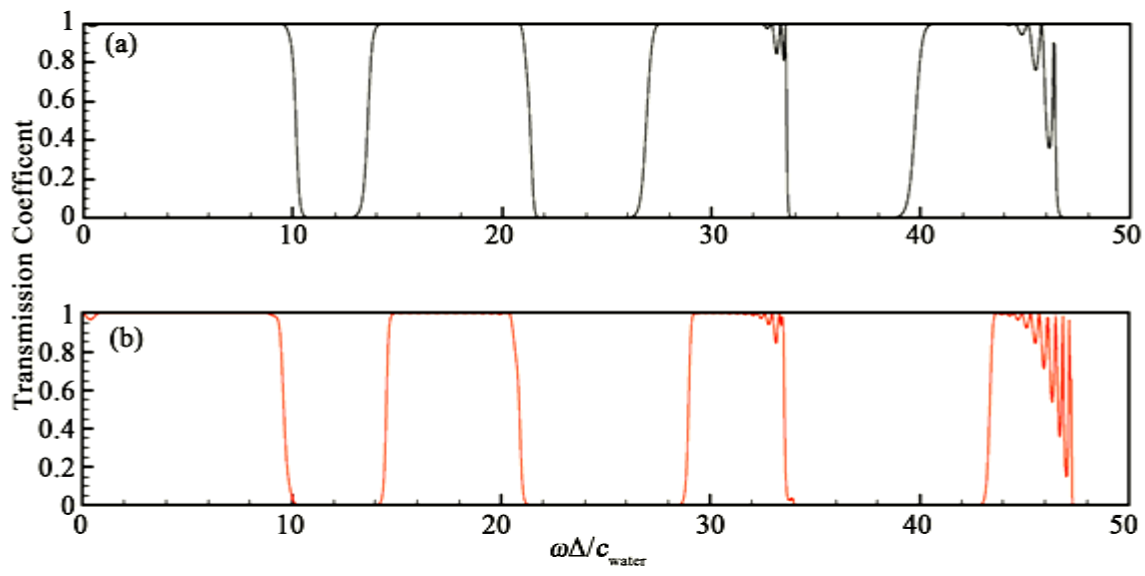

Figure 5. Transmission coefficient for a different value of $d_{Z}$ on the structure with 45 layers and for a ratio $d_{G} / d_{I}=3$.
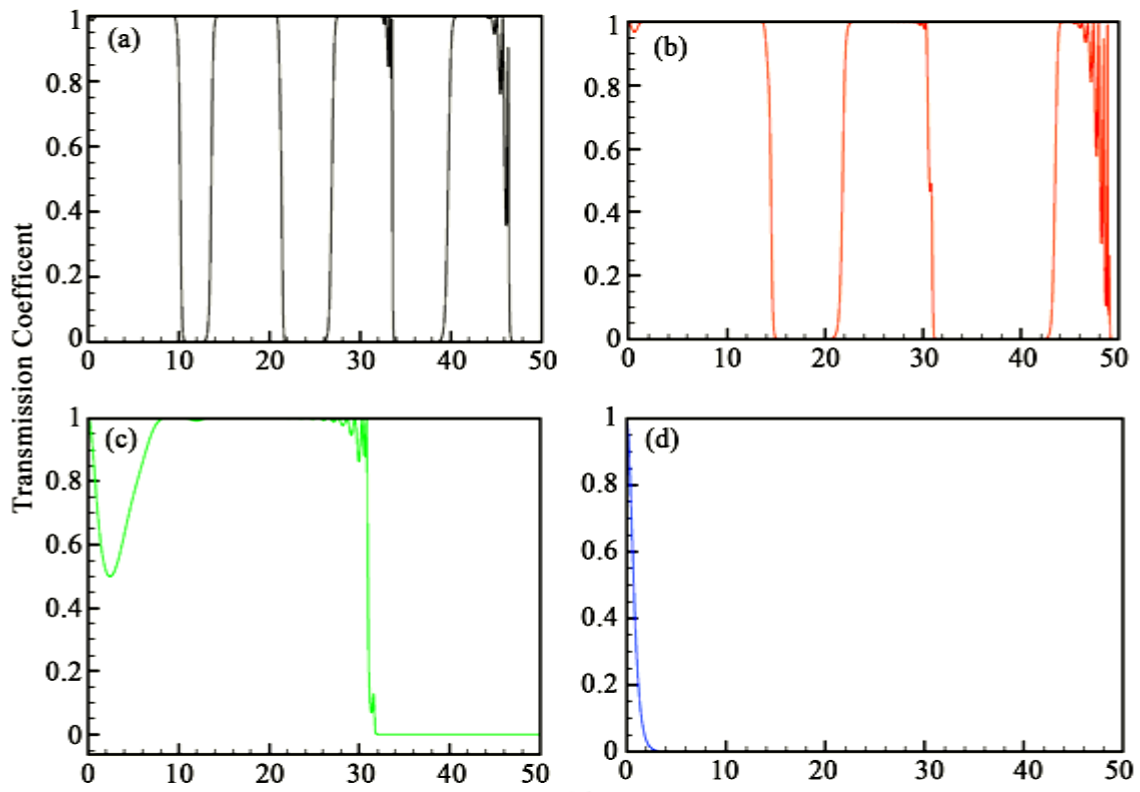

Figure 6. Transmission coefficient for oblique incidence for four values of the incident angle of the incident acoustic wave on the structure with 45 layers and for a ratio $d_{G} / d_{I}=3$.

\section{Acknowledgements}

R.A.R.V. acknowledges the support provided by Cátedras-CONACYT.

\section{References}

[1] Rayleigh, J.W.S. (1945) The Theory of Sound. Vol. 1 \& 2, Dover Publications, USA.

[2] Pupin, M.I. (1900) Wave Propagation over Non-Uniform Cables and Long Distances Air Lines. Transaction American Institute Electrical Engineering, 17, 245 307.

[3] Campbell, G.A. (1903) On Loaded Lines in Telephonic Transmission. The London Edinburgh, and Dublin Philosophical Magazine and Journal of Science, 5, 313-330. https://doi.org/10.1080/14786440309462928 
[4] Wagner, K.W. (1919) Spulen-und Kondensatorleitungen. Archiv fr Elektrotechnik, 8, 61-92. https://doi.org/10.1007/BF01597052

[5] Stewart, G.W. (1922) Theory of Acoustic Filters: The Limiting Frequencies of Transmission. Physical Review, 19, 401. https://doi.org/10.1103/PhysRev.19.401

[6] Stewart, G.W. (1922) Acoustic Wave Filters. Physical Review, 20, 528-551. https://doi.org/10.1103/PhysRev.20.528

[7] Stewart, G.W. (1923) A Variable Single Band Acoustic Wave Filter. Physical Review, 22, 502-505. https://doi.org/10.1103/PhysRev.22.502

[8] Stewart, G.W. (1924) Acoustic Wave Filters: Attenuation and Phase Factors. Physical Review, 23, 520-524. https://doi.org/10.1103/PhysRev.23.520

[9] Peacok, H.B. (1924) Predicted Transmission Curves of Acoustic Wave Filters. Physical Review, 23, 252-527. https://doi.org/10.1103/physrev.23.525

[10] Stewart, G.W. (1925) Acoustic Wave Filter: An Extension of the Theory. Physical Review, 90, 90-98. https://doi.org/10.1103/PhysRev.25.90

[11] Mason, W.P. (1927) A Study of the Regular Combination of Acoustic Elements, with Applications to Recurrent Acoustic Filters, Tapered Acoustic Filters, and Horn. Bell System Technical Journal, 6, 258-294. https://doi.org/10.1002/j.1538-7305.1927.tb01215.x

[12] Mason, W.P. (1928) The Propagation Characteristics of Sound Tubes and Acoustic Filters. Physical Review, 31, 283-295. https://doi.org/10.1103/PhysRev.31.283

[13] Lindsay, R.B. (1929) Note on the Theory of Acoustic Wave Filters. Physical Review, 37, 652-655. https://doi.org/10.1103/PhysRev.34.652

[14] Mason, W.P. (1930) The Approximate Networks of Acoustic Filters. Bell System Technical Journal, 9, 332-340. https://doi.org/10.1002/j.1538-7305.1930.tb00370.x

[15] Ganguly, A.K. and Vasell, M.O. (1973) Frequency Response of Acoustic Surface Wave Filters. Journal of Applied Physics, 44, 1072-1085. https://doi.org/10.1063/1.1662308

[16] Matthaei, G.L. (1973) Acoustic Surface-Wave Transversal Filters. IEEE Transactions on Circuit Theory, 20, 459-470. https://doi.org/10.1109/TCT.1973.1083750

[17] Potter, B.R. and Hartmann, C.S. (1977) Low Loss Surface Acoustic Wave Filters. IEEE Transactions on Parts, Hybrids, and Packaging, 13, 348-353. https://doi.org/10.1109/TPHP.1977.1135221

[18] Moore, P.A., Murray R.J., White P.D. and Garters, J.A. (1982) Surface Acoustic Wave Filters for Use in Mobile Radio. The Radio and Electronic Engineer, 52, 139144. https://doi.org/10.1049/ree.1982.0019

[19] Stokes, R.B. and Crawford, J.D. (1993) X-Band Thin Film Acoustic Filters on GaAs. IEEE Transactions on Microwave Theory and Techniques, 41, 1075-1080. https://doi.org/10.1109/22.238530

[20] Breinig, C., Dreiseitel, P., Hansler, E., Maderm A., Nitsch, B., Puder, H., Schertler, T., Schmidt, G. and Tilp, G. (1999) Acoustic Echo Control: An Application of VeryHigh-Order Adaptive Filters. IEEE Signal Processing Magazine, 16, 42-69. https://doi.org/10.1109/79.774933

[21] Mason, P., Amran, M. and Ostiguy, G. (1995) Theoretical and Experimental Study of Plane Wave Propagation in Asymmetrical Rectangular Waveguide Filters. Journal of Sound and Vibration, 179, 327-345. https://doi.org/10.1006/jsvi.1995.0020

[22] Kadota, M. and Kitamura, T. (1999) Influence of Leaky Surface Acoustic Wave Velocity of Glass Substrate on Frequency Variation of $\mathrm{ZnO} /$ Glass SAW Filters. IEEE 
Transactions on Ultrasonics, Ferroelectrics, and Frequency Control, 46, 817-822. https://doi.org/10.1109/58.775645

[23] Halmrast, T. (2000) Orchestal Time: Comb-Filter Coloration from Reflections. Journal of Sound and Vibration, 232, 53-69. https://doi.org/10.1006/jsvi.1999.2700

[24] Su, Q.-X., Kirby, P., Komuro, E., Imura, M., Zhang, Q. and Whatmore, R. (2001) Thin-Film Bulk Acoustic Resonators and Filters Using ZnO and Lead-ZirconiumTitanate Thin Films. IEEE Transactions on Microwave Theory and Techniques, 49 , 769-788.

[25] Nejade, A. and Singh, R. (2002) Flexural Intensity Measurement of Finite Plates Using Modal Spectrum Ideal Filtering. Journal of Sound and Vibration, 2561, 33 63. https://doi.org/10.1006/jsvi.2001.4198

[26] Kim, S. and Park, Y. (2004) A Direct Design Method of Inverse Filters for Multichannel 3D Sound Redering. Journal of Sound and Vibration, 278, 1196-1204.

[27] Bai, M.R. and Elliot, S.J. (2004) Preconditioning Multichannel Adaptive Filtering Algorithms Using EVD- and SVD-Based Signal Prewhitening System Decoupling. Journal of Sound and Vibration, 270, 639-655.

[28] Laje, R. and Mindlin, G.B. (2005) Modeling Source-Source and Source-Filter Acoustic Interaction in Birdsong, Physical Review E, 72, Article ID: 036218. https://doi.org/10.1103/PhysRevE.72.036218

[29] Allam, S. and Abom, M. (2005) Acoustic Modeling and Testing of Diesel Particulate Filters. Journal of Sound and Vibration, 288, 255-273.

[30] Zhou, Y.-L., Zhang, Q.-Z, Li, Z.-D. and Gan, W.-S. (2005) Analysis and DSP Implementation of an ANC System Using a Filtered-Error Neural Network. Journal of Sound and Vibration, 285, 1-25.

[31] Preumont, A., Francois, A., De Man, P., Loix, N. and Henrioulle, K. (2005) Distributed Sensors with Piezoelectric Films in Design of Spatial Filters for Structural Control. Journal of Sound and Vibration, 282, 701-712.

[32] Wen, J., Hashimoto, K.-Y., Omori, T., Yamaguchi, M., Kawachi, O. and Ueda, M. (2006) Suppression of Reflection Coefficients of Surface Acoustic Wave Filters Using Quadrature Hybrids. IEEE Transactions on Ultrasonics, Ferroelectrics, and Frequency Control, 53, 1912-1917. https://doi.org/10.1109/TUFFC.2006.124

[33] Judge, J.A., Houston, B.H., Photiadis, D.M. and Herdic, P.C. (2006) Effects of Disorder in One- and Two-Dimensional Micromechanical Resonator Arrays for Filtering. Journal of Sound and Vibration, 290, 1119-1140.

[34] Zeng, J. and de Callafon, R.A. (2006) Recursive Filter Estimation for Feed-Forward Noise Cancellation with Acoustic Coupling. Journal of Sound and Vibration, 291, 1061-1079.

[35] Allan, S. and Abom, M. (2006) Sound Propagation in an Array of Narrow Porous Channels with Applications to Diesel Particulate Filters. Journal of Sound and Vibration, 29, 882-901.

[36] Yuan, J. (2007) Adaptive Laguerre Filters for Active Noise Control. Applied Acoustics, 68, 86-96.

[37] Chang, C.-Y. and Luoh, F.-B. (2007) Enhancement of Active Noise Control Using Neural-Based Filtered-X Algorithm. Journal of Sound and Vibration, 305, 348-356.

[38] Djamaa, M.C., Ouelaa, N., Pezerat, C. and Guyader, J.L. (2007) Reconstruction of a Distributed Force Applied on a Thin Cylindrical Shell by an Inverse Method and Spatial Filtering. Journal of Sound and Vibration, 301, 560-575.

[39] Chen, K., Xu, P.-Y., Lu, J. and Xu, B.-L. (2009) An Improved Post-Filter of Acoustic 
Echo Canceller Based on Subband Implementation. Applied Acoustics, 70, 886-893.

[40] Yan, R. and Gao, R.X. (2010) Harmonic Wavelet-Based Data Filtering for Enhanced Machine Defect Identification. Journal of Sound and Vibration, 329, 3203-3217.

[41] Chivukula, V.B. and Rhoads, J.F. (2010) Microelectromechanical Bandpass Filters Based on Cyclic Coupling Architectures. Journal of Sound and Vibration, 329, 4313-4332.

[42] Zhang, H. and Pang, W. (2011) A Novel Single-Ended to Balanced Bulk Acoustic Filter for Wireless Communications. IEEE Microwave and Wireless Components Letters, 21, 347-349. https://doi.org/10.1109/LMWC.2011.2145415

[43] Pasco, Y., Robin, O., Bélanger, P., Berry, A. and Rajah, S. (2011) Multi-Input MultiOutput Feed forward Control Multi-Harmonic Gearbox Vibrations Using Parallel Adaptive Notch Filters in the Principal Component Space. Journal of Sound and Vibration, 330, 5230-5244.

[44] Sobreira-Seoane, M.A., Pérez-Cabo, D. and Jacobsen, F. (2012) The Influence of the Group Delay of Digital Filters on Acoustic Decay Measurements. Applied Acoustics, 73, 877-833.

[45] Meltaus, J., Pensala, T. and Kokkenn, K. (2012) Parametric Study of Laterally Acoustically Coupled Bulk Acoustic Wave Filters. IEEE Transactions on Ultrasonics, Ferroelectrics, and Frequency Control, 59, 2742-2751. https://doi.org/10.1109/TUFFC.2012.2515

[46] Gupta, N., Voloshinivo, V.B., Knyazev, G.A. and Kulakova, L.A. (2012) Tunable Wide-Angle Acoustic-Optic filter in Single-Crystal Tellerium. Journal of Optics, 14, Article ID: 035502. https://doi.org/10.1088/2040-8978/14/3/035502

[47] Xua, X., Herrin, D.W., Wu, T.W. and Elnady, T. (2013) Simulation of Diesel Particulate Filters in Large Exhaust Systems. Applied Acoustics, 74, 1326-1332.

[48] Yasuda, T., Wu, C., Nakagama, N. and Nagamura, K. (2013) Studies on an Automobile Muffle with the Acoustic Charactersitic of Low-Pass Filter and Helmholtz Resonator. Applied Acoustics, 74, 49-57.

[49] Wang, C., Hu, F, He, Q., Zhang, A., Liu, F. and Kong, F. (2014) De-Noising of Wayside Acoustic Signal from Train Bearings Based on Variable Digital Filtering, Applied Acoustics, 83, 127-140.

[50] Gauthier, P.-A., Gérard, A., Camier, C. and Berry, A. (2014) Acoustical Inverse Problems Regularization: Direct Definition of Filters Factors Using Signal-to-Noise Ratio. Journal of Sound and Vibration, 333, 761-773.

[51] Fromenteze, T., Decroze, C., Carsenat, D., Crunteanu, A., Chatras, M. and Passerieux, D. (2015) Passive Beam Forming Using Surface Acoustic Wave Filters. IEEE Antennas and Wireless Propagation Letters, 14, 76-79.

[52] De Oliveira, A.G., Ventura, T.M., Ganche, T.D., de Figueiredo, Jahn, O., Marques, M.I. and Schuchmann, K.L. (2015) Bird Acoustic Activity Detection Based on Morphological Filtering of the Spectrogram. Applied Acoustics, 98, 34-42.

[53] Spa, C., Rey, A. and Hernández, E. (2015) A GPU Implementation of an Explicit Compact FDTD Algorithm with a Digital Impedance Filter for Room Acoustics Applications. IEEE Transactions on Audio Speech and Language Processing, 23, 1368-1380. https://doi.org/10.1109/TASLP.2015.2434212

[54] Barberi, R., Berberi, N. and de Lima, K.F. (2015) Some Applications of the PSO for Optimization of Acoustic Filters. Applied Acoustics, 89, 62-70.

[55] Muromets, A.V., Voloshinov, V.B. and Kononnin, I.A. (2016) Transmission Characteristics of Acousto-Optic Filter Using Sectioned Transducer. Applied Acoustics, 
$112,221-225$.

[56] Červenka, M. and Bednařík, M. (2016) Acoustic Bandpass Filters Employing Shaped Resonators. Journal of Sound and Vibration, 383, 76-88.

[57] Kaizuka, T., Tanaka, N. and Nakano, K. (2016) Active Control of Sound Transmission Using Structural Modal Filters. Journal of Sound and Vibration, 381, 14-29.

[58] Lee, F.W., Lim, K.S. and Chai, H. (2016) Determination and Extraction of RayleighWaves for Concrete Cracks Characterization Based on Matched Filtering of Center of Energy. Journal of Sound and Vibration, 636, 303-315.

[59] Lee, C.H., Lee, H.R.L., Wong, K.T. and Razo, M. (2016) The Spatial-Matched-Filter Beam Pattern of a Biaxial Non-Orthogonal Velocity Sensor. Journal of Sound and Vibration, 367, 250-255.

[60] Li, H., Sun, L., Du, W., Zhou, T. and Chen, B. (2017) Multiple-Input MultipleOutput Passive Time Reserval Acoustic Communications Using Filtered Multitone Modulation. Applied Acoustics, 119, 29-38.

[61] Jiang, G., Liu, Y., Wu, Y., Xu, W., Kong, Q. and Zhang, C. (2017) Transmission and Radiation of Acoustic Oblique Incident through Tube Arrays Based on Phononic Crystal Theory. Applied Acoustics, 116, 117-126.

[62] Huang, J., Ruzzene, M. and Chen, S. (2017) Analysis of In-Plane Wave Propagation in Periodic Structures with Sierpinski-Carpet Unit Cells. Journal of Sound and Vibration, 395, 127-141.

[63] Guo, Z., Sheng, M. and Pan, J. (2017) Effect of Boundary Conditions on the BandGap Properties of Flexural Waves in a Periodic Compound Plate. Journal of Sound and Vibration, 395, 102-126.

[64] Tung, H.H. and Lee, C.P. (1996) An Energy Band-Pass Filter Using Superlattice Structures. IEEE Journal of Quantum Electronics, 32, 507-512.

https://doi.org/10.1109/3.485403

[65] Gómez, E.D.I., Domínguez-Adame, F. and Bellani, V. (1999) Electron Transport across a Gaussian Superlattice. Journal of Applied Physics, 85, 3916-3918. https://doi.org/10.1063/1.369764

[66] Arriaga, J. and Saldaña, X. (2006) Band Structure and Reflectivity of Omnidirectional Si-Based Mirrors with a Gaussian Profile Refractive Index. Journal of Applied Physics, 100, Article ID: 044911. https://doi.org/10.1063/1.2336078

[67] Yeh, P. (1988) Optical Waves in Layered Media. Wiley, New York.

[68] Shung, K.K. and Zippuro, M. (1996) Ultrasonic Transducers and Arrays. IEEE Engineering in Medicine and Biology Magazine, 6, 20-30. https://doi.org/10.1109/51.544509

[69] Zhang, R., Cao, W., Zhou, Q., Cha, J.H., Shung, K.K. and Huang, Y. (2007) Acoustic Properties of Alumina Colloidal/Polymer Nano-Composite Film on Sillicon. IEEE Transactions on Ultrasonics, Ferroelectrics and Frequency Control, 54, 467-469. https://doi.org/10.1109/TUFFC.2007.270

[70] Boumaiza, A.D., Hadjoub, Z. and Deboub, L. (1999) Porosity Effects on Different Measured Acoustic Parameters of Porous Silicon. Journal of Materials Science Letters, 18, 295-297. https://doi.org/10.1023/A:1006618920416

[71] Doghmane, M.D.A., Hadjoub, O. and Hadjoub, Z. (2007) Prediction of Elastic Parameters of Photoluminescent Porous Si. ICTON-MW, Sousse, 6-8 December 2007, $1-4$.

[72] Reinhardt, A. and Snow, P.A. (2007) Theoretical Study of Acoustic Band-Gap Structures Made of Porous Silicon. Physica Status Solidi, 204, 1528-1535. 
https://doi.org/10.1002/pssa.200674415

[73] Madrigal-Melchor, J., Enciso-Muñoz, A. and Contreras-Solorio, D.A. (2013) Acoustic Transmittance of an Aperiodic Deterministic Multilayer Structure. IOP Conference Series: Materials Science and Engineering, 45, Article ID: 012030. https://doi.org/10.1088/1757-899X/45/1/012030

[74] Barajas-Aguilar, A.H., Rodríguez-Magdaleno, K.A., Martínez-Orozco, J.C., Enciso-Muñoz, A. and Contreras-Solorio, D.A. (2013) Transmission of Electrons with Flat Passbands in Finite. IOP Conference Series: Materials Science and Engineering, 45, Article ID: 012031. https://doi.org/10.1088/1757-899X/45/1/012031

[75] Madrigal-Melchor, J., Enciso-Muñoz, A. and Contreras-Solorio, D.A. (2017) Optical Transmittance of a Multilayer Structure with Gaussian Modulation of the Refractive Index. IOP Conference Series: Materials Science and Engineering, 45, Article ID: 012032.

[76] Elmore, W.C. and Heald, M.A. (1985) Physics of Waves. Dover, New York.

[77] Brekhovskikh, L.M. (1960) Waves in Layered Media. Academic Press, New York.

[78] Kinsler, L.E., Frey, A.R., Coppens, A.B. and Sanders, J.V. (1997) Fundamentals of Acoustic. Wiley, New York.

[79] Ristic, V.M. (1983) Principles of Acoustic Devices. Wiley and Sons, Hoboken.

[80] Cao, W. and Qi, W. (1995) Plane Wave Propagation in Finite 2-2 Composites. Journal of Applied Physics, 78, 4627-4632. https://doi.org/10.1063/1.360701

\section{Submit or recommend next manuscript to SCIRP and we will provide best} service for you:

Accepting pre-submission inquiries through Email, Facebook, LinkedIn, Twitter, etc. A wide selection of journals (inclusive of 9 subjects, more than 200 journals)

Providing 24-hour high-quality service

User-friendly online submission system

Fair and swift peer-review system

Efficient typesetting and proofreading procedure

Display of the result of downloads and visits, as well as the number of cited articles

Maximum dissemination of your research work

Submit your manuscript at: http://papersubmission.scirp.org/

Or contact oja@scirp.org 